\title{
Acupuncture treatment for sleep disturbances patients: A case report with \\ inflammatory cytokine levels evaluation
}

\author{
Yun Jin Kim
}

School of Medicine, Xiamen University Malaysia Campus, Selangor, Malaysia

\section{Corresponding Author:}

Yun Jin Kim

School of Medicine, Xiamen University Malaysia Campus

Jalan Sunsuria, Bandar Sunsuria, Sepang,

Selangor 43900, Malaysia

Email: neurokim76@naver.com

\section{ABSTRACT}

Sleep disturbance is one of the more common risk factors, numerous other negative physical health outcomes, and it is defined as self-reported poor quality of sleep, short sleep duration, daytime sleepiness, and insomnia symptoms. It is a common risk factor for depression and anxiety. Here, a case of sleep disturbance that received 72 sessions of acupuncture treatment delivered is reported. After acupuncture treatment, the patient's PSQI, HDRS, and SAS score decreased, as well as the plasma TNF- $\alpha$, and IL- 6 being reduced. No adverse effects were observed. The clinical studies findings provide evidence supporting the acupuncture aimed at the cause of sleep disturbance symptoms can also be useful.

\section{Key Words}

Sleep disturbance, acupuncture, inflammatory cytokine

\section{Implications for Practice:}

\section{What is known about this subject?}

Sleep disturbance is one of the more common risk factors, numerous other negative physical health outcomes.

\section{What new information is offered in this case study?}

This case study offered acupuncture management for sleep disturbances patients and also had shown the inflammatory cytokine levels evaluation.

3. What are the implications for research, policy, or practice?

All patients with sleep disturbances can be managed with acupuncture treatment.

\section{Background}

Acupuncture is a form of traditional \& complementary medicine in which thin acupuncture needles are inserted into the body. It is most used commonly for pain relief, though it is also used to treat a wide range of conditions. ${ }^{1}$ Its positive effect has been shown in a variety of neurodegenerative, psychosomatic diseases, cerebral or peripheral ischemia, and Parkinson's disease. Also applied in various diseases involving psychosomatic status, such as anxiety, depression, and sleep disturbances. ${ }^{2}$

Sleep disturbance is defined as self-reported poor quality of sleep, short sleep duration, daytime sleepiness, and insomnia symptoms Annually over 50 million adults in the US suffer from sleep disturbances. ${ }^{3}$ Despite the psychological, physical, and financial burden of sleep disturbance, it remains an under-diagnosed and poorly understood condition. In primary care and the specialist hospital setting, sleep disturbance is often inadequately discussed and effectively treated. It is large gap between clinical practice guidelines and treating sleep disturbance and current primary clinical care system. ${ }^{4}$ Cytokines as signalling molecules are involved in the regulated in part by humoral substances in sleep disturbance. Cytokines are well characterized for roles in sleep regulation. Scientific experimental approaches have been used to discover and characterize cytokines and sleep regulatory substances. It is knowledge of cytokine sleep mechanisms that provide additional support for the hypotheses that sleep serves a synaptic-connectivity function. ${ }^{5}$ William et al. ${ }^{6}$ studied that cytokines significantly associated with psychological 
symptoms, their founded IL-6 was significantly associated with depression, and somewhat more strongly with the somatic symptoms of depression compared to cognitive/affective symptoms.

In this case report, we present the one clinical case report with sleep disturbance that was treated and underwent 72 sessions of acupuncture. After acupuncture treatment response as evaluated, in addition to Pittsburgh Sleep Quality Index (PSQI), Hamilton Depression Rating Scale (HDRS), Zung Self-Rating Anxiety Scale (SAS) included Plasma TNF- $\alpha$, and IL-6.

\section{Case details}

A 50-year-old Malaysian-Chinese female primary school teacher with difficulty in falling and initiating sleep, and day time depression or anxiety about it being very difficult to sleep at night, had been ongoing for three years She already had consulted a neurologist, psychologist, and family doctors but had become worse during the previous eight months, and was referred to our clinic. She had the complaints of trouble with difficulty in falling and staying asleep, her spending one or more hours before being able to sleep but one or two hours afterwards becoming awake, and unable to continue to sleep. Daytime she felt depression, or anxiety, and cannot focus upon her work. She already had taken medication Zopiclone $7.5 \mathrm{mg}$ per day. Before referred to our clinic, she discussed with her neurologist and psychologist ceasing her current medication, and doing acupuncture treatment instead. After acupuncture treatment, she had a better sleep, and decreased depression, and anxiety symptoms.

Our institution does not require ethical approval for reporting individual cases or case series. But, we have verbal informed consent obtained from the patient for their anonymized information to be published in this case report article.

\section{Acupuncture treatment}

The acupoints are used for sleep disturbance: Xinshu (BL 15), Shenshu (BL 23), PiShu (BL 20), Ganshu (BL 18), Qimen (LR 14), Taichong(LR 3), and Zusanli (ST 36). The acupoints used in this case study were selected based on previous scientific reports of acupuncture for the treatment of sleep disturbance patients. ${ }^{7,8}$

The disposable sterilized acupuncture needles used size $0.3 \mathrm{~mm} \times 40 \mathrm{~mm}$ (Hansol Medical Co. Korea. Reg No.: 437) and were inserted into each acupoints. After insertion and the needling sensation of obtaining Qi (de qi), every 10 minutes a needle manipulation involving twirling the needle for one minute was done, and the needles were kept in place for 30 minutes without electrostimulator's stimulation. The acupuncture treatment was given three times per week for total 72 sessions (six months).

\section{Evaluation}

Before acupuncture treatment, the patient had measured her Pittsburgh Sleep Quality Index (PSQI), Hamilton Depression Rating Scale (HDRS), Zung Self-Rating Anxiety Scale (SAS) and plasma TNF- $\alpha$, and IL- 6 , and the values were used to establish a baseline. During acupuncture sessions, the patient's scores and plasma tests were monitored bimonthly. The plasma samples were investigated at BP Clinical Lab \& Diagnostic Centre (M) Sdn. Bhd in the Taman Sutera Danga branch, Johor, Malaysia, and the PQSI, HDRS, and SAS was determined at Cheng De TCM Specialist, Johor, Malaysia.

Table 1 showed pre- and post- acupuncture treatment PSQI score. Usually, a PSQI score of $>5$ means with poor sleep quality, and $\leq 5$ is mean of good sleep quality. After six months of acupuncture treatment, the PSQI score decreased in compare to the baseline.

Table 2 showed pre- and post- acupuncture treatment TNF$\alpha$ and II- 6 level. After six months of acupuncture treatment, the TNF- $\alpha$ and II- 6 level reduced in comparison to the baseline. And, Figure 1 presents pre- and post- acupuncture treatment HDRS and SAS. After six months of acupuncture treatment, the HDRS and SAS decreased in comparison to the baseline.

\section{Discussion}

Currently review articles have shown many diseases have sleep disturbance or are directly associated with sleep problems, acupuncture offers useful benefits, often resulting in improved sleep quality, reduced depression and anxiety. ${ }^{9}$ Evidence is accumulating that acupuncture is moderately helpful for both these psychiatric problems. Many clinical positive case reports and trials confirming benefit from acupuncture therapy for sleep disturbance, so acupuncture appears to be effective in treatment of sleep disturbance. Cochrane review was able to analyse 33 RCTs and concluded acupuncture used as an adjunct to routine therapy may improve sleep quality. ${ }^{10}$ Several related reports suggested that sleep disturbance is associated with elevations in systemic levels of inflammation, like IL- 6 and TNF- $\alpha$. Healthy normal sleepers subjected to total and partial sleep restriction showed significant elevated results in inflammatory activity compared to an undisturbed sleep 
conditions. Furthermore comparison to depressed symptoms, depression patients displayed higher nocturnal levels of IL-6, a difference that is partially accounted for by a longer time to sleep onset in the depression patients. ${ }^{11-13}$

In acupuncture prescription, the Back-Shu acupoints of Xinshu (BL 15), Shenshu (BL 23), PiShu (BL 20), Ganshu (BL 18), are tonified and nourished the Heart, Kidney, Bladder, and Liver, regulates the Qi, removes Blood stasis, Moves Qi locally and along the meridian pathway. Qimen (LR 14) indicated regulates Liver Qi and Liver Blood, cools the Blood, disperses accumulations, harmonies the Liver and the Stomach. Taichong(LR 3) effects that spreads Liver Qi, Clears the head, nourished Liver Blood and Liver Yin, and Zusanli (ST 36) actions for tonifies Qi and Yang, nourished the Blood and Yin, claims the Shen. ${ }^{14}$

In this clinical case report, we investigated the acupuncture treatment in sleep disturbance, and we found acupuncture can produce reduced and decreased PSQI, HDRS, and SDS score, and also plasma TNF- $\alpha$ and IL-6 levels. Based on this case study, the authors recommended that acupuncture is safe and clinical effective therapy for sleep disturbance. However, future studies are needed with large groups for investigation, as well as ascertaining any beneficial treatment effect that might be achieved by including additional acupuncture treatment.

Regarding safety for acupuncture treatment, Vincent in a BMJ editorial stated that acupuncture therapy in skilled hands is "one of the safer forms of medical intervention". ${ }^{15}$ A later prospective observational study from Germany of over 220,000 patients reported 2.2 per cent of adverse events due to acupuncture treatment, including two pneumothoraxes. Otherwise all adverse effects were minor, like bleeding and bruising. The report concluded, "Acupuncture provided by physicians is a relatively safe treatment". ${ }^{16}$

\section{Conclusion}

Acupuncture has been used very effectively to treat sleep disturbance, without any of the side effects of prescription. Improved sleep is only one of the benefits reported by people who have used acupuncture to treat sleep disturbance and related mental disorder. ${ }^{17}$ Acupuncture therapy can assist sleep disturbance patients with safe treatment.

\section{References}

1. Ernst E, Lee MS, Choi TY. Acupuncture: does it alleviate pain and are there serious risks? A review of reviews.
Pain. 2011;152(4):755-764.

2. Chung KF, Yeung WF, Yu YM, et al. Acupuncture for residual insomnia associated with major depressive disorder: a placebo- and sham- controlled, subject- and assessor-blind, randomized trial. J Clin Psychiatry. 2015;76(6):752-760.

3. Prather $\mathrm{H}$, Creighton $\mathrm{A}$, Sorenson $\mathrm{C}$, et al. Anxiety and insomnia in young- and middle-aged adult hip pain patients with and without femoroacetabular impingement and developmental hip dysplasia. PM R. 2017.

4. Hohagen F, Rink K, Käppler C, et al. Prevalence and treatment of insomnia in general practice. A longitudinal study. Eur Arch Psychiatry Clin Neurosci. 1993;242(6):329-336.

5. Krueger JM, Rector DM, Churchill L. SLEEP AND CYTOKINES. Sleep Med Clin. 2007;2(2):161-169.

6. William B, Barry R, Kristen T, et al. Depression, cytokines, and pancreatic cancer. Psychology. 2014;23(3):339-345.

7. Li T, Ha L, Cao F, et al. Experience summary of professor WANG Fuchun's "Zhenjing Anshen" acupuncture method for Insomnia. Zhongguo Zhen Jiu. 2015;35(11):1159-1162.

8. Hai $Y$, Zhagn $W$, Liu $E$, et al. Factors influencing acupuncture for Insomnia. Zhongguo Zhen Jiu. 2015;35(10):1057-1059.

9. Huang W, Kutner N, Bliwise DL. A systematic review of the effects of acupuncture in treating insomnia. Sleep Md Rev. 2009;13(1):73-104.

10. Cheuk DKL, Yeung WF, Chung KF, et al. Acupuncture for insomnia. Cochrane Database Syst Rev. 2012;9:CD005472.

11. Solarz DE, Mullington JM, Meier-Ewert HK. Sleep, inflammation and cardiovascular disease. Front Biosci. 2012;4:2490-2501.

12. Motivala SJ, Sarfatti A, Olmos L, et al. Inflammatory markers and sleep disturbance in major depression. Psychosom Med. 2005;67(2):187-194.

13. Vgontzas AN, Zoumakis E, Bixier EO, et al. Adverse effects of modest sleep restriction on sleepiness, performance, and inflammatory cytokines. J Clin Endocrino Metab. 2004;89(5):2119-2126.

14. Xu TB. Clinical observation on treatment of insomnia with puncturing Back-Shu acupoints. J Acupunct Tuina Sci. 2013;11(3):165-168.

15. Vincent $C$. The safety of acupuncture: acupuncture is safe in the hands of competent practitioners. BMJ. 2001;323(7311):467-468.

16. Witt CM, Pach D, Brinkhaus B, et al. Safety of acupuncture: results of a prospective observational study with 229,230 patients and introduction of a 
medical information and consent form. Forsch. Komplementmed. 2009;16(2):91-97.

17. Chung KF, Yeung WF, Yu BY, et al. Acupuncture with or without combined auricular acupuncture for insomnia: a randomised, waitlist-controlled trail. Acupunct Med. 2017.

\section{ACKNOWLEDGEMENTS}

We thank Ms Yun Jeong Seo for her oversight of this manuscript and all the participants for their time and cooperation.

\section{PEER REVIEW}

Not commissioned. Externally peer reviewed.

\section{CONFLICTS OF INTEREST}

The authors declare that they have no competing interests.

\section{FUNDING}

None

\section{PATIENT CONSENT}

The authors, $\operatorname{Kim} Y J$, declare that:

1. They have obtained written, informed consent for the publication of the details relating to the patient(s) in this report.

2. All possible steps have been taken to safeguard the identity of the patient(s).

3. This submission is compliant with the requirements of local research ethics committees.

Figure 1: Pre- and post- acupuncture treatment HDRS and SAS

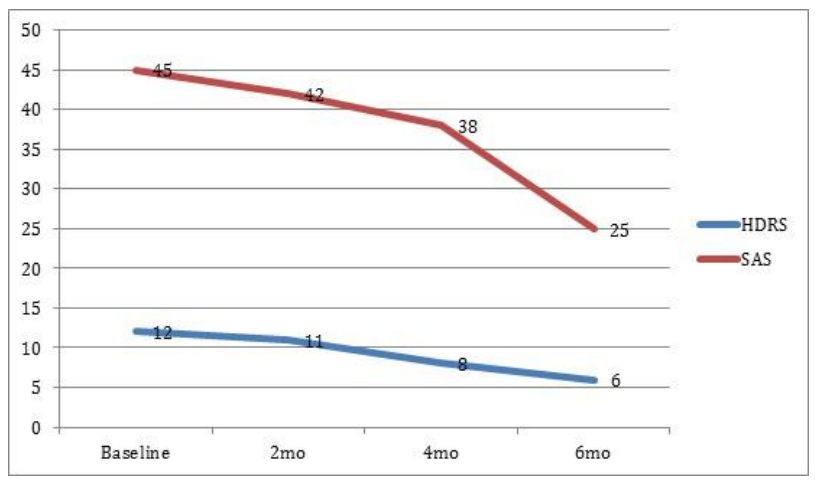

A lower score indicated a better outcome HDRS = Hamilton Depression Rating Scale SAS = Zung Self-Rating Anxiety Scale
Table 1: Pre- and post- acupuncture treatment PSQI Score results

\begin{tabular}{|l|l|l|l|l|}
\hline Component Score (0-3) & Baseline & $\mathbf{2 m o}$ & $\mathbf{4 m o}$ & $\mathbf{6 m o}$ \\
\hline Subjective sleep quality & 3 & 3 & 2 & 1 \\
\hline Sleep latency & 2 & 2 & 1 & 1 \\
\hline Sleep duration & 3 & 2 & 2 & 1 \\
\hline Habitual sleep efficiency & 3 & 3 & 2 & 1 \\
\hline Sleep disturbances & 2 & 2 & 2 & 1 \\
\hline $\begin{array}{l}\text { Use of sleeping } \\
\text { medication }\end{array}$ & 0 & 0 & 0 & 0 \\
\hline Daytime dysfunction & 3 & 3 & 2 & 1 \\
\hline Total PSQI Score & 16 & 15 & 11 & 6 \\
\hline A lower score indicated & better outcome & &
\end{tabular}

A lower score indicated a better outcome

PSQI=Pittsburgh Sleep Quality Index

Table 2: Pre- and post- acupuncture treatment plasma TNF- $\alpha$ and II- 6 level

\begin{tabular}{|l|l|l|l|l|}
\hline & Baseline & 2mo & 4mo & 6mo \\
\hline TNF- $\alpha$ (ng/ml) & 2.37 & 2.21 & 1.57 & 1.28 \\
\hline IL-6 (pg/ml) & 189.63 & 159.14 & 132.79 & 112.32 \\
\hline
\end{tabular}

A lower score indicated a better outcome 